\title{
The Costs and Healthcare Resource Utilization Associated with Anticholinergic Burden in Long-Stay Nursing Home Residents with Overactive Bladder in the US
}

\author{
Satabdi Chatterjee ${ }^{1} \cdot$ David Walker $^{2} \cdot$ Tomomi Kimura $^{3} \cdot$ Rajender R. Aparasu $^{1}$ (D)
}

Accepted: 13 June 2021 / Published online: 13 July 2021

(c) The Author(s) 2021

\begin{abstract}
Background Overactive bladder $(\mathrm{OAB})$ is a prevalent condition commonly treated with anticholinergic medications. The extent to which anticholinergic burden is associated with costs and healthcare resource use (HCRU) in the long-stay nursing home (LSNH) setting is currently unknown.

Objectives This research evaluated the impact of anticholinergic burden on HCRU and related costs among LSNH residents with OAB.

Methods This was a cohort study based on 2013-2015 Minimum Data Set-linked Medicare claims data involving LSNH residents aged $\geq 65$ years with $\mathrm{OAB}$ and having Parts A, B and D coverage 6 months pre- and $\geq 12$ months post-nursing home admission date (index date). Cumulative anticholinergic burden was determined using the Anticholinergic Cognitive Burden scale and defined daily dose. Direct medical costs related to HCRU were examined. HCRU included inpatient, outpatient, emergency room (ER), and physician office visits. Costs and HCRU associated with levels of anticholinergic burden were evaluated using generalized linear models.

Results A total of 123,308 LSNH residents with OAB were included in this study. Most residents (87.2\%) had some level (12.8\%, none; $18.0 \%$, low; $41.9 \%$, moderate; and $27.3 \%$, high) of cumulative anticholinergic burden. Results indicate that all types of resource utilization were higher among those with any level of anticholinergic burden than those with no burden. The outpatient, ER, and physician costs tended to be higher with increasing anticholinergic burden.

Conclusions Costs and HCRU patterns reflected increasing trends with anticholinergic burden. Targeted efforts towards reducing anticholinergic burden among LSNH residents with OAB may result in decreases in costs and HCRU.
\end{abstract}

\section{Introduction}

Overactive bladder $(\mathrm{OAB})$ comprises a constellation of urinary symptoms typically characterized by urgency, with or without urge incontinence, and accompanied by frequency and nocturia $[1,2]$. It is particularly common among older

Rajender R. Aparasu

rraparasu@uh.edu

1 Department of Pharmaceutical Health Outcomes and Policy, College of Pharmacy, University of Houston, Health and Biomedical Sciences Building 2, Office 4052, 4849 Calhoun Road, Houston, TX 77204-5047, USA

2 Medical Affairs, U.S., Astellas Pharma Global Development, Inc., Northbrook, IL, USA

3 Advanced Informatics and Analytics, Real World Data and Evidence, Astellas US LLC, Northbrook, IL, USA adults [3] and as a result poses a significant burden in nursing home populations [4]. Estimates of the prevalence of $\mathrm{OAB}$, specifically within the nursing home setting are scarce, although one study conducted among Medicare beneficiaries ascertained from administrative claims data that $36 \%$ of long-term residents had a diagnosis of OAB [5]. Previous studies have indicated that $\mathrm{OAB}$ poses a substantial economic burden to the US, $24 \%$ of which ( $\$ 2.85$ billion in 2000) occurs in the institutional setting [6]. Based on this economic burden of $\mathrm{OAB}$, it can be estimated that the current total costs associated with $\mathrm{OAB}$ in the institutional setting were approximately $\$ 20$ billion in 2020 [7].

Currently, the management of OAB in the nursing home setting consists of both pharmacological and non-pharmacological strategies. Recommended non-pharmacological strategies include behavioral techniques such as prompted 


\section{Key Points for Decision Makers}

The findings of this study indicate that all types of resource utilization were higher among long-stay nursing home (LSNH) residents with overactive bladder (OAB) and any level of anticholinergic burden compared with those with no anticholinergic burden.

Healthcare costs tended to be higher among $\mathrm{OAB}$ patients with higher anticholinergic burden.

Targeted efforts towards reducing anticholinergic burden among LSNH residents with OAB may result in decreases in healthcare resource utilization and costs.

and timed voiding, habit retraining, pelvic floor muscle exercises, and bladder training; implementation of these strategies are guided by a resident's mobility and cognitive status [8]. Research indicates that these non-pharmacological strategies are successful within an OAB population, and it is therefore recommended that these strategies be implemented prior to the initiation of pharmacotherapy $[9,10]$. Among residents with dementia and those who are relatively immobile, behavioral strategies may be less successful and the use of pads and diapers may be preferred. Both behavioral strategies and the use of pads and diapers avoid contributing to polypharmacy among patients; however, it may add to the economic burden of the condition $[11,12]$. OAB pharmacotherapy is primarily comprised of antimuscarinic agents, which are part of the class of oral anticholinergic medications [13]; however, anticholinergics are associated with several central and peripheral adverse effects. Additionally, the safety and tolerability issues associated with antimuscarinics have resulted in suboptimal adherence and persistence to these medications among OAB patients [14, 15]. Furthermore, cumulative exposure to anticholinergic medications ('anticholinergic burden') has been associated with a greater risk of adverse effects [16-19]. A recent retrospective analysis using administrative Medicare claims data found that anticholinergic burden was present in almost two-thirds of beneficiaries [16]. The consequences of anticholinergic burden among this vulnerable population range from impairments in physical performance and the ability to perform activities of daily living (ADLs) to an increased risk of dementia [17-19]. Furthermore, given the safety concerns, the most recent version of the American Geriatrics Society Beers Criteria includes the recommendation to avoid drugs with strong anticholinergic properties [20].
Several studies have shown that anticholinergic burden increased healthcare resource utilization and costs, although these findings were largely derived from outpatient populations [21-23]. Retrospective analyses using US electronic medical record (EMR) data revealed that among elderly individuals (age 65 years and older), increases in anticholinergic burden were associated with greater odds of emergency room (ER) visits and both inpatient and outpatient hospitalizations [21]. Similar findings have been observed among other global settings, including an analysis of older outpatients in New Zealand, where increases in anticholinergic burden were also associated with greater odds of inpatient and outpatient visits [24]. Finally, a population-based study of elderly individuals in Finland revealed that level of anticholinergic burden was associated with a greater risk of hospitalization [23]. Although there is extensive anticholinergic use and associated anticholinergic burden among nursing home residents $[25,26]$, the extent to which anticholinergic burden is associated with healthcare resource use (HCRU) and costs in a nursing home setting is currently unknown.

We recently evaluated anticholinergic burden among long-stay nursing home (LSNH) residents and found that nearly $90 \%$ of those with $\mathrm{OAB}$ were exposed to varying levels of burden, two-thirds of whom demonstrated moderate-to-high levels. Level of exposure (no, low, moderate, or high) was ascertained by evaluating a drug's anticholinergic activity and patient-specific dosing [25]. Given the anticholinergic burden and impact of this burden on healthcare, it is particularly important to assess the healthcare resource utilization and costs associated with anticholinergic burden among LSNH to understand the resource implications of managing OAB. Thus, the objective of this study was to evaluate the impact of anticholinergic burden on health care use and related costs in LSNH residents with OAB.

\section{Methods}

\subsection{Data Source}

This was a retrospective cohort study using Minimum Data Set (MDS)-linked Medicare claims data from January 2013 to December 2015, involving Parts A, B and D. The MDS is a standardized assessment and is performed within nursing homes that are eligible for Medicare or Medicaid funding. At a minimum, data on long-term residents are collected on a quarterly basis and includes information on a variety of outcomes, including, but not limited to, patient functioning, cognition, mobility, behavioral symptoms, diagnoses, and medications [27]. Medicare Standard Analytical Files (SAFs) are restricted to claims submitted by Fee-for-Service enrollees and include specific files on inpatients, outpatients, 
skilled nursing homes, carriers and durable medical equipment, as well as MedPAR, the Denominator file, Vital Stats and Part D files [28, 29]. SAFs do not include patients from Medicare managed care plans. This study was approved under exempt category by the University of Houston Committee for the Protection of Human Subjects as this was a retrospective study involving de-identified data.

\subsection{Study Design and Study Cohort}

Residents were considered to be long-stay if they had at least one nursing home stay of at least 101 consecutive days [30]. Additional criteria for inclusion into the study were age 65 years or older at the time of nursing home admission (index date), and having Parts A, B and D coverage 6 months before, and a minimum of 12 months following, the index date. In addition to the Parts $\mathrm{A}, \mathrm{B}$ and $\mathrm{D}$ coverage requirements, residents were required to have at least 280 days of nursing home stay following the index date in order to assess anticholinergic exposure over a 6-month follow-up period. This is because Medicare Part D data were used to assess anticholinergic use, and medications used during the first 100 days following admission are generally covered by Medicare Part A. Finally, patients were required to have at least one medication claim during the 6-month period starting on the 101st day of admission. The presence of OAB was based on inpatient and/or outpatient International Classification of Diseases Ninth/Tenth Revision (ICD-9/10) diagnosis codes or a claim for an $\mathrm{OAB}$-specific medication based on prescription fill records or CPT codes during the study period. Specific diagnostic and medication codes, as well as a schematic of patient enrollment and follow-up, are available in the study by Chatterjee et al. [25].

\subsection{Anticholinergic Exposure}

Cumulative anticholinergic burden was calculated using previously published methods that adjust for a drug's anticholinergic activity along with patient-specific dosing [18, 31]. In this study, anticholinergic medication use was assessed for each patient over a 6-month period, starting from the 101st day of admission, and was based on the medications listed in the Anticholinergic Cognitive Burden (ACB) scale [32]. A multistep process was subsequently used to ascertain patient-specific dosing. This first included the standardization of differences in medication dosing using the World Health Organization's (WHO) defined daily dose (DDD) in order to obtain the standardized daily dose (SDD) [31, 33]. It should be noted that the DDD does not necessarily correspond to the recommended dose or prescribed daily dose (PDD) [33]. Next, the SDD for each patient was multiplied by the corresponding ACB scale score to obtain the drug-specific standardized daily anticholinergic exposure
(SDACE). A summated standardized daily anticholinergic exposure (SumSDACE) was obtained by totaling the SDACE values for each day of the study period. The daily SumSDACE values were then totaled over the 6-month follow-up period starting from the 101st day after admission in order to obtain cumulative exposure [31]. Previously published cut-off values of cumulative SumSDACE scores were used to classify patients with no (0), low (1-89), moderate (90-499), or high ( $\geq 500)$ anticholinergic burden [34]. Additional detail regarding the calculation of cumulative anticholinergic exposure can be found in the study by Chatterjee et al. [25].

\subsection{Measures}

The outcomes of this study were healthcare resource utilization and costs, according to the levels of anticholinergic burden. HCRU included inpatient, outpatient, ER, and physician office visits that occurred during the 6 months of follow-up from the 101st day of nursing home admission through day 280. Inpatient utilization was defined as the number of allcause hospital admissions during this period. Direct medical costs related to HCRU during the 6-month follow-up period were tabulated for each patient.

\subsection{Statistical Analysis}

Descriptive statistics were used to summarize patient demographic and clinical characteristics by levels of cumulative anticholinergic burden (no, low, moderate, and high). Multiple regression models were subsequently developed to evaluate HCRU and costs associated with cumulative anticholinergic burden. HCRU with skewed count data was analyzed using negative binomial distributed generalized linear models (GLMs) with a log-link function. Coefficients for negative binomial models were computed as incidence rate ratios (IRR). All models were adjusted for demographics, function (ADLs), and clinical characteristics by using multiple propensity score adjustment [26, 35]. Finally, costs were analyzed using gamma-distributed log-link GLMs and adjusted to 2019 US dollar (US\$) values using the consumer price index (CPI). Coefficients for gamma-distributed cost models were computed as cost ratios.

Sensitivity analyses were employed in order to examine the robustness of study findings based on the definitions of certain exposure and outcome variables. Specifically, inclusion criteria were modified so that OAB patients were only identified by ICD-9/10 codes for OAB. Furthermore, the first 100 days of stay (Part D coverage) were considered as part of the calculation of cumulative anticholinergic burden. All regression analyses were repeated to examine the impact of these changes on the findings. 


\section{Results}

\subsection{Baseline Characteristics}

A total of $124,345 \mathrm{LSNH}$ residents with OAB were identified during the study period. The mean age was $82.6( \pm$ 8.28 ) years, $72.7 \%$ were female, and $87.3 \%$ were non-Hispanic White. The most common comorbidities overall were hypertension, fluid and electrolyte disorders, and depression. The majority of residents received antidepressant medications and were dependent with regard to performing ADLs. A total of 123,308 (99.2\%) residents had at least one medication claim during the 6-month period starting from the 101st day of admission, and formed the analytical cohort for multivariable analyses. Most of these residents (87.2\%) had some level of anticholinergic burden during the 6-month period starting from the 101st day of admission based on the cumulative SumSDACE. The cumulative SumSDACE scores were used to classify patients into the following categories of anticholinergic burden: $12.8 \%$ with no cumulative anticholinergic burden, $18.0 \%$ with low cumulative anticholinergic burden, $41.9 \%$ with moderate cumulative anticholinergic burden, and $27.3 \%$ with high cumulative anticholinergic burden (Table 1; electronic supplementary Table 1).

\subsection{Healthcare Resource Utilization}

Regarding healthcare resource utilization, the mean number of outpatient visits increased with the levels of anticholinergic burden. Residents in the no burden group had an average of 4.82 (SD 5.76) outpatient visits, those with low burden had 5.75 (6.58) outpatient visits, those with moderate burden had 6.77 (7.52) outpatient visits, and those with high burden had 7.81 (8.48) outpatient visits. The percentage of those with one or more visits also increased with levels of burden $(82.77 \%, 86.67 \%, 89.23 \%$, and $90.99 \%$ for those with no, low, moderate, and high levels of burden, respectively). While the mean number of inpatient hospitalizations did not differ by level of burden, a higher percentage of those with any anticholinergic burden had one or more hospital admissions than those with no burden $(15.12 \%, 24.38 \%, 25.35 \%$, and $25.00 \%$ for those with no, low, moderate, and high levels of burden, respectively). Likewise, the mean number of physician visits did not differ according to levels of burden. However, the percentage of those with one or more visits increased with levels of burden $(40.90 \%, 46.43 \%, 51.79 \%$, and $57.91 \%$ for those with no, low, moderate, and high levels of burden, respectively). Finally, the mean number of ER visits increased slightly with level of burden. Specifically, the mean number of visits was 0.39 (SD 0.82), 0.59 (SD 1.02 ), 0.65 (SD 1.10), and 0.67 (SD 1.12) for those with no, low, moderate, and high levels of burden, respectively. The percentage of those with one or more emergency visits also increased with levels of burden $(25.81 \%, 36.12 \%$, $37.83 \%$, and $38.68 \%$ for those with no, low, moderate, and high levels of burden, respectively) (Table 2). Finally, results from the negative binomial regression analyses indicate that after adjusting for covariates, all types of resource utilization were higher among those with any level of anticholinergic burden (low, moderate, or high) than those with no burden (Table 3).

\subsection{Healthcare Costs}

In terms of mean healthcare costs, expenditures for those with moderate/high levels of burden were higher in all types of HCRU than those with no/low levels of burden. The per-person annual costs associated with outpatient visits increased from $\$ 1889$ for those in the no burden group to $\$ 2780$ for those with high burden. Costs associated with physician visits increased from $\$ 84$ to $\$ 143$ for those with no burden and those with high burden, respectively, while costs associated with ER visits increased from \$179 to \$299 for those with no burden and those with high burden, respectively. For inpatient hospitalizations, mean costs were lowest among those with no burden (\$2161), but those with low and moderate burden (\$3850 and \$3862, respectively) had slightly higher costs than those with high burden (\$3700) (Table 4).

Results from the propensity score-adjusted regression analysis indicated that patients with low burden incurred $6 \%$ higher inpatient costs than patients with no burden in the adjusted analysis (IRR 1.06, 95\% confidence interval [CI] 1.01-1.11). For high versus no burden, admission costs were $5 \%$ lower in the high burden group (IRR $0.95,95 \% \mathrm{CI}$ 0.91-0.99), and for moderate versus no burden, there was no difference (IRR 0.99, 95\% CI 0.96-1.04). For adjusted outpatient department costs, all low, moderate, and high burden groups were significantly higher than the no burden group. The IRR for the low burden group was 1.09 (95\% CI 1.05-1.13), the IRR for the moderate burden group was 1.10 (95\% CI 1.06-1.13), and the IRR for the high burden group was 1.11 (95\% CI 1.08-1.15). For adjusted physician office visits, high burden patients had significantly higher costs than those with no burden (IRR $1.05,95 \%$ CI 1.02-1.08). Furthermore, there was no significant difference between low burden versus no burden (IRR 1.01, 95\% CI 0.97-1.04) or moderate burden versus no burden (IRR 1.03, 95\% CI 0.99-1.06). Finally, for adjusted ER visits, low and moderate burden were associated with higher costs than no burden. The IRR for the low burden group was 1.07 (95\% CI 1.03-1.11) and the IRR for the moderate burden group was 1.04 (95\% CI 1.01-1.08). However, for the high burden 
Table 1 Baseline characteristics of elderly nursing home residents with OAB, categorized by levels of cumulative anticholinergic burden

Elderly long-stay NH patients with OAB having prescription records during 101-280 days of follow-up [ $N=123,308]$

Characteristics

$\begin{array}{ll}\text { No burden (score 0) } & \text { Low burden (score } \\ {[n=15,801](12.8 \%)} & 1-89)[n=22,215] \\ & (18.0 \%)\end{array}$

Moderate burden (score

90-499) $[n=51,668]$

(41.9\%)

Age, years

65-74 [ $n=24,067]$

$75-84[n=43,269]$

$85+[n=55,964]$

Sex

Male [ $n=33,511]$

Female [ $n=89,437$ ]

Race/ethnicity ${ }^{\mathrm{a}}$

Non-Hispanic White [ $n=107,659$ ]

Non-Hispanic Black $[n=10,785]$

Hispanics [ $n=1802$ ]

Other [ $n=2847]$

Marital status ${ }^{\mathrm{b}}$

Married [ $n=29,429]$

Unmarried [ $n=93,138]$

Medicare/Medicaid dual eligible

[ $n=56,966$ ]

Region $^{\text {a }}$

South $[n=44,987]$

Northeast $[n=26,586]$

Midwest $[n=39,969]$

West $[n=11,750]$

Others $[n=10]$

Urban/Rural

Rural [ $n=35,152]$

Urban $[n=88,156]$

Baseline medication use [ $n(\%)]$

Antidepressants

Diuretics

$\beta$-Blockers

Calcium channel blockers

Anticonvulsants

ACE inhibitors

Antipsychotics

Antiparkinson agents

$\alpha$-Blockers

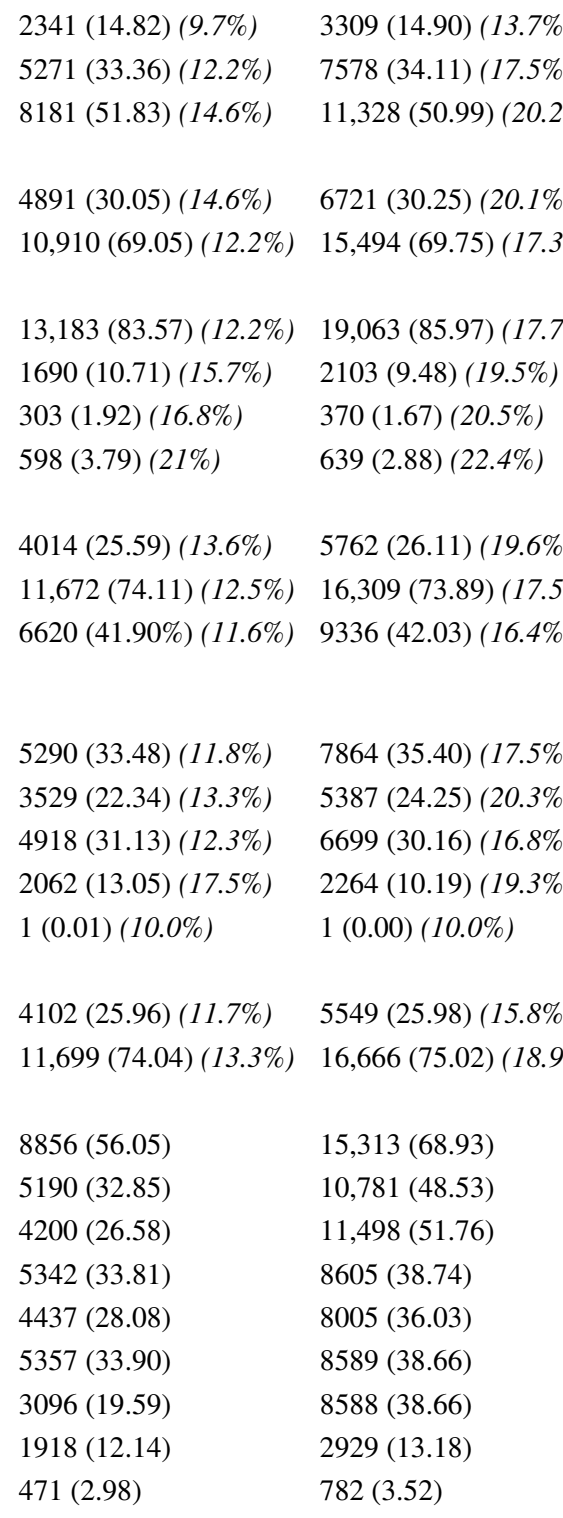

High burden (score $\geq 500)[n=33,624]$ (27.3\%)

\section{0 (25.96) (36.0\%) \\ $12,170(36.19)(28.0 \%)$}

12,724 (37.84) (22.7\%)

7966 (23.69) (23.8\%)

25,658 (76.31) (28.7\%)

30,191 (89.94) (28.0\%)

$2499(7.44)(23.2 \%)$

352 (1.05) (19.5\%)

525 (1.56) (18.4\%)

7302 (21.84) (24.8\%)

26,125 (78.16) (28.0\%)

17,082 (50.80) (30.0\%)

$12,608(37.50)(28.0 \%)$
$6556(19.50)(24.7 \%)$
$11,607(34.52)(29.0 \%)$
$2847(8.47)(24.2 \%)$
$3(0.01)(30.0 \%)$

10,731 (31.91) (30.5\%)

22,893 (68.09) (26.0\%)

$27,072(80.51)$

$24,280(72.21)$

22,116 (65.77)

15,565 (46.29)

$16,830(50.05)$

14,774 (43.94)

14,739 (43.83)

6709 (19.95)

1349 (4.01)

Italicized values indicate the percentage based on the row total; non-italicized percentage values refer to the percentage of patients in each anticholinergic burden category. Baseline Elixhauser comorbidities can be found in electronic supplementary Table 6

$A C E$ angiotensin-converting enzyme, $M D S$ Minimum Data Set, $N H$ nursing home, $O A B$ overactive bladder

${ }^{a}$ Based on enrollment files and includes missing data. Denominator for the percentage calculation is those who have non-missing data

${ }^{\mathrm{b}}$ Based on MDS admission assessment and includes missing data. Denominator for the percentage calculation is those who have non-missing data 
Table 2 Healthcare resource utilization by levels of cumulative anticholinergic burden in patients with OAB

\begin{tabular}{|c|c|c|c|c|c|}
\hline Utilization & No burden & Low burden & Moderate burden & High burden & $p$ value \\
\hline \multicolumn{6}{|l|}{ Hospitalization utilization } \\
\hline One or more hospital admissions & $2389(15.12)$ & $5415(24.38)$ & $13,097(25.35)$ & $8407(25.00)$ & $<0.0001$ \\
\hline \multicolumn{6}{|l|}{ Hospitalization distribution } \\
\hline 0 & $13,412(84.88)$ & $16,800(75.62)$ & $38,571(74.65)$ & $25,217(75.00)$ & \multirow[t]{4}{*}{$<0.0001$} \\
\hline 1 & $1815(11.49)$ & $3839(17.28)$ & $9179(17.77)$ & $5884(17.50)$ & \\
\hline 2 & $440(2.78)$ & $1097(4.94)$ & $2662(5.15)$ & $1728(5.14)$ & \\
\hline$>2$ & $134(0.85)$ & $479(2.16)$ & $1256(2.43)$ & $795(2.36)$ & \\
\hline \multicolumn{6}{|l|}{ Number of admissions } \\
\hline Mean (SD) number of admissions & $0.35(0.72)$ & $0.37(0.76)$ & $0.36(0.76)$ & $0.36(0.75)$ & \multirow[t]{2}{*}{$<0.0001$} \\
\hline Median (IQR) & $0(0-0)$ & $0(0-0)$ & $0(0-0)$ & $0(0-0)$ & \\
\hline \multicolumn{6}{|l|}{ OD visits } \\
\hline One or more visits & $13,079(82.77)$ & $19,254(86.67)$ & $46,104(89.23)$ & 30,595 (90.99) & $<0.0001$ \\
\hline \multicolumn{6}{|l|}{ Distribution of $O D$ visits } \\
\hline 0 & $2722(17.23)$ & $2961(13.33)$ & $5564(10.77)$ & $3029(9.01)$ & \multirow[t]{4}{*}{$<0.0001$} \\
\hline 1 & $1908(12.08)$ & $2387(10.74)$ & $4750(9.19)$ & $2680(7.97)$ & \\
\hline 2 & $1933(12.23)$ & $2486(11.19)$ & $5273(10.21)$ & $3063(9.11)$ & \\
\hline$>2$ & $9238(58.46)$ & $14,381(64.74)$ & $36,081(69.83)$ & $24,852(73.91)$ & \\
\hline Mean (SD) number of OD visits & $4.82(5.76)$ & $5.75(6.58)$ & $6.77(7.52)$ & $7.81(8.48)$ & $<0.0001$ \\
\hline Median (IQR) & $3(1-5)$ & $4(2-8)$ & $5(2-9)$ & $5(2-10)$ & \\
\hline \multicolumn{6}{|l|}{ Physician visits } \\
\hline One or more visits & $6462(40.90)$ & $10,315(46.43)$ & $26,760(51.79)$ & $19,470(57.91)$ & $<0.0001$ \\
\hline \multicolumn{6}{|l|}{ Distribution of physician visits } \\
\hline 0 & $9339(59.10)$ & $11,900(53.57)$ & $24,908(48.21)$ & $14,154(42.09)$ & \multirow[t]{4}{*}{$<0.0001$} \\
\hline 1 & $2436(15.42)$ & $3894(17.53)$ & $9124(17.66)$ & $5996(17.83)$ & \\
\hline 2 & $1431(9.06)$ & $2159(9.72)$ & $5753(11.13)$ & $4081(12.14)$ & \\
\hline$>2$ & $2595(16.42)$ & $4262(19.19)$ & $11,883(23.00)$ & $9393(27.94)$ & \\
\hline Mean (SD) number of physician visits & $1.23(2.41)$ & $1.42(2.54)$ & $1.69(2.81)$ & $2.05(3.11)$ & $<0.0001$ \\
\hline Median (IQR) & $0(0-0)$ & $0(0-0)$ & $1(0-2)$ & $1(0-3)$ & \\
\hline \multicolumn{6}{|l|}{ ER visits utilization } \\
\hline One or more ER visits & $4079(25.81)$ & $8023(36.12)$ & $19,548(37.83)$ & $13,006(38.68)$ & $<0.0001$ \\
\hline \multicolumn{6}{|l|}{ Distribution of ER visits } \\
\hline 0 & $11,722(74.19)$ & $14,192(63.88)$ & $32,120(62.17)$ & $20,618(61.32)$ & \multirow[t]{6}{*}{$<0.0001$} \\
\hline 1 & $2774(17.56)$ & $4967(22.36)$ & $11,748(22.74)$ & $7689(22.87)$ & \\
\hline 2 & $836(5.29)$ & $1828(8.23)$ & $4550(8.81)$ & $3121(9.28)$ & \\
\hline$>2$ & $469(2.97)$ & $1228(5.53)$ & $3250(6.29)$ & $2196(6.53)$ & \\
\hline Mean (SD) number of ER visits & $0.39(0.82)$ & $0.59(1.02)$ & $0.65(1.10)$ & $0.67(1.12)$ & \\
\hline Median & $0(0-0)$ & $0(0-0)$ & $0(0-0)$ & $0(0-0)$ & \\
\hline
\end{tabular}

Data are expressed as $n(\%)$ unless otherwise specified

$E R$ emergency room, $I Q R$ interquartile range, $O A B$ overactive bladder, $O D$ outpatient department, $S D$ standard deviation

$P$ values $\leq 0.05$ are considered significant

group, there was no difference compared with those with no burden (IRR 0.99, 95\% CI 0.96-1.03) (Table 5). Finally, results from the sensitivity analyses were consistent with all trends observed in the primary analyses (electronic supplementary Tables 2-5).

\section{Discussion}

To the authors' knowledge, this is the first US study to evaluate health care utilization and costs associated with anticholinergic burden among a nationally representative 
Table 3 Health care utilization among long-stay nursing home residents with OAB: results from propensity score-adjusted negative binomial regression analysis

\begin{tabular}{llll}
\hline Utilization & $\begin{array}{l}\text { Cumulative } \\
\text { anticholinergic } \\
\text { burden }\end{array}$ & $\begin{array}{l}\text { Unadjusted } \\
\text { IRR }(95 \% \mathrm{CI})\end{array}$ & $\begin{array}{l}\text { Adjusted } \\
\text { IRR }(95 \% \mathrm{CI})\end{array}$ \\
\hline $\begin{array}{c}\text { Hospital } \\
\text { admission }\end{array}$ & No & Reference & Reference \\
& Low & $1.73(1.64-1.89)$ & $1.41(1.34-1.49)$ \\
& Moderate & $1.83(1.75-1.92)$ & $1.32(1.26-1.38)$ \\
OD visits & High & $1.80(1.72-1.89)$ & $1.16(1.10-1.22)$ \\
& No & Reference & Reference \\
& Low & $1.19(1.16-1.22)$ & $1.11(1.09-1.14)$ \\
Mhysician visits & Moderate & $1.40(1.37-1.43)$ & $1.19(1.16-1.22)$ \\
& High & $1.62(1.58-1.66)$ & $1.27(1.24-1.30)$ \\
& No & Reference & Reference \\
& Low & $1.15(1.11-1.20)$ & $1.08(1.04-1.13)$ \\
& Moderate & $1.33(1.38-1.43)$ & $1.13(1.09-1.17)$ \\
ER visits & High & $1.67(1.61-1.73)$ & $1.22(1.18-1.27)$ \\
& No & Reference & Reference \\
& Low & $1.52(1.46-1.58)$ & $1.30(1.25-1.35)$ \\
& Moderate & $1.65(1.59-1.72)$ & $1.29(1.24-1.34)$ \\
& High & $1.71(1.64-1.77)$ & $1.22(1.17-1.34)$ \\
\hline
\end{tabular}

$C I$ confidence interval, $E R$ emergency room, $I R R$ incidence rate ratio, $O A B$ overactive bladder, $O D$ outpatient department

sample of $\mathrm{OAB}$ patients in the long-term care facility setting. Previous studies have mainly focused on outpatient settings and have found that anticholinergic burden increased healthcare resource utilization and costs [21-23]. This study involving LSNH residents with OAB found that overall, healthcare resource utilization and costs increased with anticholinergic burden in the US. The largest differences in health care utilization and costs were observed when those with no anticholinergic burden were compared with those with any level of burden (low, moderate, and high).

Despite differences in study populations, these findings are largely in line with previous observations that healthcare resource utilization among community-dwelling adults with $\mathrm{OAB}$ increased with higher levels of anticholinergic burden [36]. Lozano-Ortega et al. found that among commercially insured $\mathrm{OAB}$ patients in the US, approximately $65 \%$ of individuals had some level of anticholinergic burden [36]. Significantly greater healthcare resource utilization was observed among those with any level of anticholinergic burden (low, moderate, or high) versus those with no burden. Indeed, the relationships reported by LozanoOrtega et al. between level of anticholinergic burden and healthcare costs were more consistently linear than the relationships in the present study, where costs and utilization were at times observed to peak with moderate levels of burden [36]. However, although there was variability in healthcare costs across anticholinergic burden groups in the present study, healthcare costs were generally higher among those with higher anticholinergic burden. Furthermore, the results of this study are also consistent with research conducted among a general older adult population, where it was observed that the relationship between level of anticholinergic burden and healthcare costs persisted subsequent to adjustment for patient comorbidities [21, 37]. A study of 3344 community-dwelling older adults in the US found that increasing levels of anticholinergic burden were also associated with higher costs in analyses that adjusted for comorbid conditions [21]. Furthermore, a study using Taiwanese administrative claims found that after adjusting for comorbidities, level of anticholinergic burden was associated with an increased risk of ER visits and all-cause hospitalization [37].

The study findings suggest the need to reduce anticholinergic burden and its associated healthcare impact. A systematic review by Nakham et al. indicated that while exposure could be successfully reduced, the impact of a reduction in exposure has not been adequately evaluated [38]. However, studies using the AGS Beers criteria to guide reductions in polypharmacy among individuals in long-term care can provide some evidence regarding the potential impact of anticholinergic burden on healthcare costs $[39,40]$. Another study found that after an intervention to reduce polypharmacy, a reduction in anticholinergics accounted for approximately $10 \%$ of the total decrease in healthcare costs [39]. Thus, given the high healthcare resource utilization and costs associated with anticholinergic burden, efforts to reduce the levels of anticholinergic burden could result in decreased utilization and costs, specifically among LSNH residents with OAB. Additionally, the use of pads and diapers would avoid contributing to anticholinergic burden in the LSNH population with OAB $[11,12]$; however, it is unclear whether their use would result in a net reduction economic burden and warrants further investigation.

The limitations of this study include those that are inherent to administrative claims data. This includes issues related to the accuracy of medication recording; for example, whether or not a prescribed medication was actually taken cannot be verified, which could potentially result in the overestimation of level of burden. Conversely, as the use and coverage of over-the-counter medications vary by state and are not captured by Medicare, they would not be captured in this study. This may have resulted in an underestimation of burden. Although the DDD helps to standardize drug consumption, it does not reflect recommended dose or PDD [33]. Other issues include the 
Table 4 Health care costs (2019 US\$) among long-stay nursing home residents with OAB

\begin{tabular}{|c|c|c|c|c|c|c|}
\hline \multirow[t]{2}{*}{ Unit } & \multirow[t]{2}{*}{ Variable } & \multicolumn{4}{|c|}{ Levels of cumulative anticholinergic burden } & \multirow[t]{2}{*}{$p$ value } \\
\hline & & No burden & Low burden & Moderate burden & High burden & \\
\hline \multirow[t]{12}{*}{ US\$ } & \multicolumn{6}{|c|}{ Hospital admission } \\
\hline & Mean $( \pm$ SD $)$ & $2160.68(7235.65)$ & $3849.85(10,192.41)$ & $3862.09(9973.69)$ & $3700.45(9449.97)$ & \multirow[t]{2}{*}{$<0.0001$} \\
\hline & Median (IQR) & $0(0-0)$ & $0(0-0)$ & $0(0-2521.90)$ & $0(0-0)$ & \\
\hline & \multicolumn{6}{|l|}{ OD visits } \\
\hline & Mean $( \pm \mathrm{SD})$ & $1888.97(3334.54)$ & $2293.73(3804.29)$ & $2536.39(3955.25)$ & $2779.85(4045.19)$ & \multirow[t]{2}{*}{$<0.0001$} \\
\hline & Median (IQR) & $752.04(74.58-2330.64)$ & $1057.52(152.98-899.31)$ & $1268.54(239.53-260.77)$ & $1488.93(334.48-3607.00)$ & \\
\hline & \multicolumn{6}{|c|}{ Physician visits } \\
\hline & Mean $( \pm$ SD) & $83.88(171.95)$ & $98.81(184.31)$ & $119.41(208.24)$ & $143.42(225.60)$ & \multirow[t]{2}{*}{$<0.0001$} \\
\hline & Median (IQR) & $0(0-100.61)$ & $0(0-130.90)$ & $21.85(0-161.97)$ & $61.92(0-197.30)$ & \\
\hline & \multicolumn{6}{|l|}{ ER visits } \\
\hline & Mean $( \pm S D)$ & $179.44(449.94)$ & $283.18(576.64)$ & $297.58(590.22)$ & $299.06(582.63)$ & \multirow[t]{2}{*}{$<0.0001$} \\
\hline & Median (IQR) & $0(0-98.24)$ & $0(0-383.15)$ & $0(0-399.11)$ & $0(0-400.86)$ & \\
\hline
\end{tabular}

$E R$ emergency room, $I Q R$ interquartile range, $O A B$ overactive bladder, $O D$ outpatient department, $S D$ standard deviation, $U S \$$ United States dollars

possibility of medical coding errors within the database, although the potential impact of this on the study findings cannot be assessed. While the database used in this study provides a fairly robust range of variables for analyses, there are likely many unmeasurable variables that impact healthcare use and costs. Finally, the findings represent an association between anticholinergic burden and health care use and costs but not causation.

Table 5 Health care costs (2019 US\$) in patients with OAB: results from regression analysis using gamma distribution

\begin{tabular}{llll}
\hline Utilization & $\begin{array}{l}\text { Cumulative } \\
\text { anticholinergic } \\
\text { burden }\end{array}$ & $\begin{array}{l}\text { Unadjusted } \\
\text { IRR }(95 \% \text { CI })\end{array}$ & $\begin{array}{l}\text { Adjusted } \\
\text { IRR }(95 \% \mathrm{CI})\end{array}$ \\
\hline $\begin{array}{c}\text { Hospital } \\
\text { admission }\end{array}$ & No & Reference & Reference \\
& Low & $1.10(1.06-1.15)$ & $1.06(1.01-1.11)$ \\
& Moderate & $1.06(1.02-1.11)$ & $0.99(0.96-1.04)$ \\
OD visits & High & $1.03(0.99-1.08)$ & $0.95(0.91-0.99)$ \\
& No & Reference & Reference \\
& Low & $1.16(1.12-1.30)$ & $1.09(1.05-1.13)$ \\
& Moderate & $1.24(1.20-1.28)$ & $1.10(1.06-1.13)$ \\
Physician visits & High & $1.33(1.29-1.38)$ & $1.11(1.08-1.15)$ \\
& No & Reference & Reference \\
& Low & $1.03(0.99-1.07)$ & $1.01(0.97-1.04)$ \\
& Moderate & $1.11(1.08-1.14)$ & $1.03(0.99-1.06)$ \\
& High & $1.19(1.16-1.23)$ & $1.05(1.02-1.08)$ \\
ER visits & No & Reference & Reference \\
& Low & $1.11(1.07-1.15)$ & $1.07(1.03-1.11)$ \\
& Moderate & $1.13(1.09-1.17)$ & $1.04(1.01-1.08)$ \\
& High & $1.13(1.09-1.16)$ & $0.99(0.96-1.03)$ \\
& & & \\
& & &
\end{tabular}

$C I$ confidence interval, $E R$ emergency room, IRR incidence rate ratio, $O A B$ overactive bladder, $O D$ outpatient department, US\$ United States dollars
Regarding the patient population, these data are representative of Medicare Fee-For-Service enrollees and therefore may not be applicable to those covered by Medicare managed care plans. Furthermore, patients who were in the nursing home for an extended length of time prior to 2013 may have been excluded from the study as a result of the inclusion criteria. It is unknown to what extent these patients may have differed from those who were more recently admitted. Moreover, the analysis is based on data from 2013 to 2015, and the medication use and healthcare utilization patterns may not reflect current practice. More research is needed using recent data to evaluate changes in long-term care.

\section{Conclusions}

In this large, representative study of LSNH residents with $\mathrm{OAB}$ in the US, healthcare utilization and cost patterns reflected increasing trends with cumulative anticholinergic burden. Specifically, all types of resource utilization were higher among those with any level of anticholinergic burden than those with no burden. Although there was variability in healthcare costs across anticholinergic burden groups, healthcare costs tended to be higher among OAB patients with higher anticholinergic burden. Targeted efforts towards reducing anticholinergic burden among LSNH residents with $\mathrm{OAB}$ may result in decreases in healthcare resource utilization and costs. 
Supplementary Information The online version contains supplementary material available at https://doi.org/10.1007/s41669-021-00281-8.

\section{Declarations}

Funding This study was funded by Astellas Pharma Global Development. Medical writing/editorial support was provided by Alexis Mickle from Broadstreet HEOR and funded by the study sponsor.

Conflict of interest David Walker and Tomomi Kimura are employees of Astellas and may own Astellas stock. Rajender Aparasu reports grants from Incyte and Novartis, outside the submitted work. Satabdi Chatterjee has no conflicts of interest to declare.

Availability of Data and Material Data are not available due to the Centers for Medicare and Medicaid Services (CMS) data use restrictions for research identifiable files. The datasets generated and/or analyzed during the current study are available from the corresponding author on reasonable request. Researchers may request access to anonymized participant-level data, trial-level data, and protocols from Astellas sponsored clinical trials at http://www.clinicalstudydatarequest.com. For the Astellas criteria on data sharing see https://clinicalstudydatareq uest.com/Study-Sponsors/Study-Sponsors-Astellas.aspx.

Code Availability Not applicable.

Author Contributions All authors contributed to the study conception and design. Data analysis was performed by RRA and SC. All authors contributed to the writing of the manuscript, and read and approved the final manuscript.

Ethics approval Not applicable.

Consent to participate Not applicable.

Consent for publication Not applicable.

Open Access This article is licensed under a Creative Commons Attribution-NonCommercial 4.0 International License, which permits any non-commercial use, sharing, adaptation, distribution and reproduction in any medium or format, as long as you give appropriate credit to the original author(s) and the source, provide a link to the Creative Commons licence, and indicate if changes were made. The images or other third party material in this article are included in the article's Creative Commons licence, unless indicated otherwise in a credit line to the material. If material is not included in the article's Creative Commons licence and your intended use is not permitted by statutory regulation or exceeds the permitted use, you will need to obtain permission directly from the copyright holder. To view a copy of this licence, visit http://creativecommons.org/licenses/by-nc/4.0/.

\section{References}

1. Abrams P, Andersson KE, Apostolidis A, Birder L, Bliss D, Brubaker $\mathrm{L}$, et al. 6th international consultation on incontinence. Recommendations of the International Scientific Committee: evaluation and treatment of urinary incontinence, pelvic organ prolapse and faecal incontinence. Neurourol Urodyn. 2018;37(7):2271-2.

2. Eapen RS, Radomski SB. Review of the epidemiology of overactive bladder. Res Rep Urol. 2016;8:71-6.

3. Sexton CC, Coyne KS, Thompson C, Bavendam T, Chen C-I, Markland A. Prevalence and effect on health-related quality of life of overactive bladder in older Americans: results from the epidemiology of lower urinary tract symptoms study. J Am Geriatr Soc. 2011;59(8):1465-70.

4. Sura S, Shiozawa A, Ng D, Aparasu RR. Higher resource utilization and costs in long-term nursing home residents with overactive bladder: a retrospective study of medicare beneficiaries. J Am Med Dir Assoc. 2021;22(6):1300-6.

5. Sura SD, Aparasu RR. Puk19 overactive bladder patients in the nursing homes: resident characteristics and treatment pattern. Value Health. 2019;22:S384.

6. Hu TW, Wagner TH, Bentkover JD, LeBlanc K, Piancentini A, Stewart WF, et al. Estimated economic costs of overactive bladder in the United States. Urology. 2003;61(6):1123-8.

7. Ganz ML, Smalarz AM, Krupski TL, Anger JT, Hu JC, WittrupJensen KU, et al. Economic costs of overactive bladder in the United States. Urology. 2010;75(3):526-32 (532.e1-18).

8. Holroyd-Leduc JMLC, Tannenbaum C. Practical management of urinary incontinence in the long-term care setting. Ann Longterm Care. 2006;14(2)

9. Zarowitz BJ, Allen C, O'Shea T, Tangalos E, Berner T, Ouslander JG. Clinical burden and nonpharmacologic management of nursing facility residents with overactive bladder and/or urinary incontinence. Consult Pharm. 2015;30(9):533-42.

10. Tran K, Levin RM, Mousa SA. Behavioral intervention versus pharmacotherapy or their combinations in the management of overactive bladder dysfunction. Adv Urol. 2009;2009:345324.

11. Huion A, Decalf V, Kumps C, De Witte N, Everaert K. Smart diapers for nursing home residents with dementia: a pilot study. Acta Clin Belg. 2019;74(4):258-62.

12. Macdiarmid SA. Maximizing the treatment of overactive bladder in the elderly. Rev Urol. 2008;10(1):6-13.

13. Lightner Deborah J, Gomelsky A, Souter L, Vasavada SP. Diagnosis and treatment of overactive bladder (non-neurogenic) in adults: AUA/SUFU guideline amendment 2019. J Urol. 2019;202(3):558-63.

14. Yeowell G, Smith P, Nazir J, Hakimi Z, Siddiqui E, Fatoye F. Real-world persistence and adherence to oral antimuscarinics and mirabegron in patients with overactive bladder (OAB): a systematic literature review. BMJ Open. 2018;8(11):e021889.

15. Dhaliwal P, Wagg A. Overactive bladder: strategies to ensure treatment compliance and adherence. Clin Interv Aging. 2016;11:755-60.

16. Niznik J, Zhao X, Jiang T, Hanlon JT, Aspinall SL, Thorpe J, et al. Anticholinergic prescribing in medicare part $\mathrm{D}$ beneficiaries residing in nursing homes: results from a retrospective cross-sectional analysis of medicare data. Drugs Aging. 2017;34(12):925-39.

17. Landi F, Russo A, Liperoti R, Cesari M, Barillaro C, Pahor M, et al. Anticholinergic drugs and physical function among frail elderly population. Clin Pharmacol Ther. 2007;81(2):235-41.

18. Gray SL, Anderson ML, Dublin S, Hanlon JT, Hubbard R, Walker R, et al. Cumulative use of strong anticholinergics and incident dementia: a prospective cohort study. JAMA Intern Med. 2015;175(3):401-7.

19. Fox C, Richardson K, Maidment ID, Savva GM, Matthews FE, Smithard D, et al. Anticholinergic medication use and cognitive impairment in the older population: the medical research council cognitive function and ageing study. J Am Geriatr Soc. 2011;59(8):1477-83.

20. By the American Geriatrics Society Beers Criteria Update Expert P. American Geriatrics Society 2019 Updated AGS Beers Criteria ${ }^{\circledR}$ for potentially inappropriate medication use in older adults. J Am Geriatr Soc. 2019;67(4):674-94.

21. Campbell NL, Perkins AJ, Bradt P, Perk S, Wielage RC, Boustani MA, et al. Association of anticholinergic burden with cognitive impairment and health care utilization among a 
diverse ambulatory older adult population. Pharmacotherapy. 2016;36(11):1123-31.

22. Salahudeen MS, Duffull SB, Nishtala PS. Anticholinergic burden quantified by anticholinergic risk scales and adverse outcomes in older people: a systematic review. BMC Geriatr. 2015;15:31.

23. Gnjidic D, Hilmer SN, Hartikainen S, Tolppanen AM, Taipale $\mathrm{H}$, Koponen $\mathrm{M}$, et al. Impact of high risk drug use on hospitalization and mortality in older people with and without Alzheimer's disease: a national population cohort study. PLoS One. 2014;9(1):e83224.

24. Salahudeen MS, Hilmer SN, Nishtala PS. Comparison of anticholinergic risk scales and associations with adverse health outcomes in older people. J Am Geriatr Soc. 2015;63(1):85-90.

25. Chatterjee S, Walker D, Kimura T, Aparasu RR. Prevalence and factors associated with cumulative anticholinergic burden among older long-stay nursing home residents with overactive bladder. Drugs Aging. 2021;38(4):311-26.

26. Chatterjee S, Mehta S, Sherer JT, Aparasu RR. Prevalence and predictors of anticholinergic medication use in elderly nursing home residents with dementia: analysis of data from the 2004 National Nursing Home Survey. Drugs Aging. 2010;27(12):987-97.

27. Centers for Medicare and Medicaid Services. Long term care minimum data set (MDS) 3.0—data documentation. 2020. https:// www.resdac.org/cms-data/files/mds-3.0/data-documentation. Accessed 29 Oct 2020.

28. Centers for Medicare and Medicaid Services. Medicare standard analytical files: identifiable data files. http://www.cms.hhs.gov/ IdentifiableDataFiles/02_StandardAnalyticalFiles.asp. Accessed 29 Oct 2020.

29. Centers for Medicare and Medicaid Services. Research Data Assistance Center (ResDAC): available CMS data. Centers for Medicare and Medicaid Services; 2020. https://www.resdac.org. Accessed 29 Oct 2020.

30. Wei YJ, Simoni-Wastila L, Zuckerman IH, Brandt N, Lucas JA. Algorithm for identifying nursing home days using medicare claims and minimum data set assessment data. Med Care. 2016;54(11):e73-7.

31. Lozano-Ortega G, Johnston KM, Cheung A, Wagg A, Campbell NL, Dmochowski RR, et al. A review of published anticholinergic scales and measures and their applicability in database analyses. Arch Gerontol Geriatr. 2020;87:103885.
32. Boustani M, Campbell N, Munger S, Maidment I, Fox C. Impact of anticholinergics on the aging brain: a review and practical application. Aging Health. 2008;4(3):311-20.

33. WHO Collaborating Centre for Drug Statistics Methodology. Guidelines for ATC classification and DDD assignment 2020. Oslo: WHO; 2019.

34. Szabo SM, Gooch K, Schermer C, Walker D, Lozano-Ortega G, Rogula B, et al. Association between cumulative anticholinergic burden and falls and fractures in patients with overactive bladder: US-based retrospective cohort study. BMJ Open. 2019;9(5):e026391.

35. Spreeuwenberg MD, Bartak A, Croon MA, Hagenaars JA, Busschbach JJ, Andrea $\mathrm{H}$, et al. The multiple propensity score as control for bias in the comparison of more than two treatment arms: an introduction from a case study in mental health. Med Care. 2010;48(2):166-74.

36. Lozano-Ortega G, Schermer CR, Walker DR, Szabo SM, Rogula B, Deighton AM, et al. Fall/fracture-related healthcare costs and their association with cumulative anticholinergic burden in people with overactive bladder. Pharmacoecon Open. 2021;5(1):45-55.

37. Hsu WH, Wen YW, Chen LK, Hsiao FY. Comparative associations between measures of anti-cholinergic burden and adverse clinical outcomes. Ann Fam Med. 2017;15(6):561-9.

38. Nakham A, Myint PK, Bond CM, Newlands R, Loke YK, Cruickshank $\mathrm{M}$. Interventions to reduce anticholinergic burden in adults aged 65 and older: a systematic review. J Am Med Dir Assoc. 2020;21(2):172-80.e5.

39. Kojima G, Bell C, Tamura B, Inaba M, Lubimir K, Blanchette PL, et al. Reducing cost by reducing polypharmacy: the polypharmacy outcomes project. J Am Med Dir Assoc. 2012;13(9):818.e11-5.

40. Trygstad TK, Christensen D, Garmise J, Sullivan R, Wegner S. Pharmacist response to alerts generated from Medicaid pharmacy claims in a long-term care setting: results from the North Carolina polypharmacy initiative. J Manag Care Pharm. 2005; 11(7):575-83. 